\title{
Molecular modelling and pharmacophore elucidation study of the Classical Swine Fever virus helicase as a promising pharmacological target
}

The Classical Swine Fever virus (CSFV) is a major pathogen of livestock and belongs to the flaviviridae viral family. Even though there aren't any verified zoonosis cases yet, the outcomes of CSFV epidemics have been devastating to local communities. In an effort to shed light to the molecular mechanisms underlying the structural and drug design potential of the viral helicase, the three dimensional structure of CSFV helicase has been modeled, using conventional homology modelling techniques and the crystal structure of the Hepatitis C virus (HCV) as template. The established structure of the CSFV helicase has been in silico evaluated for its viability using a repertoire of in silico tools. The ultimate goal of this study is to introduce the 3D conformation of the CSFV helicase as a reliable structure that may be used as the designing platform for de novo, structure-based drug design experiments. In this direction using the modeled structure of CSVF helicase, a 3D pharmacophore was designed. The pharmacophore comprises of a series of key characteristics that molecular inhibitors must satisfy in order to achieve maximum predicted affinity for the given enzyme. Overall, invaluable insights and conclusions are drawn from this structural study of the CSFV helicase, which may provide the scientific community with the founding plinth in the fight against CSFV infections through the perspective of the CSFV helicase as a potential pharmacological target. Notably, to date no antiviral agent is available against the CSFV nor is expected soon. Subsequently, there is urgent need for new modern and state-of-the-art antiviral strategies to be developed. 


\section{Molecular modelling and pharmacophore elucidation study of the}

2 Classical Swine Fever virus helicase as a promising

3 pharmacological target

4

5 Dimitrios Vlachakis, Sophia Kossida*

6 Bioinformatics \& Medical Informatics Team, Biomedical Research Foundation, Academy of Athens, Soranou Efessiou 7 4, Athens 11527, Greece

$23{ }^{*}$ Correspondence to:

24 Sophia Kossida,

25 Bioinformatics \& Medical Informatics Team, Biomedical Research Foundation, Academy of Athens,

26 Soranou Efessiou 4, Athens 11527, Greece

27 Tel: + 302106597 199, Fax: +30 2106597545

28 E-mail: skossida@bioacademy.gr 


\section{Abstract}

2 The Classical Swine Fever virus (CSFV) is a major pathogen of livestock and belongs to

3 the flaviviridae viral family. Even though there aren't any verified zoonosis cases yet, the

4 outcomes of CSFV epidemics have been devastating to local communities. In an effort

5 to shed light to the molecular mechanisms underlying the structural and drug design 6 potential of the viral helicase, the three dimensional structure of CSFV helicase has 7 been modeled, using conventional homology modelling techniques and the crystal 8 structure of the Hepatitis C virus (HCV) as template. The established structure of the 9 CSFV helicase has been in silico evaluated for its viability using a repertoire of in silico

10 tools. The ultimate goal of this study is to introduce the 3D conformation of the CSFV

11 helicase as a reliable structure that may be used as the designing platform for de novo,

12 structure-based drug design experiments. In this direction using the modeled structure

13 of CSVF helicase, a 3D pharmacophore was designed. The pharmacophore comprises

14 of a series of key characteristics that molecular inhibitors must satisfy in order to

15 achieve maximum predicted affinity for the given enzyme. Overall, invaluable insights

16 and conclusions are drawn from this structural study of the CSFV helicase, which may

17 provide the scientific community with the founding plinth in the fight against CSFV

18 infections through the perspective of the CSFV helicase as a potential pharmacological

19 target. Notably, to date no antiviral agent is available against the CSFV nor is expected

20 soon. Subsequently, there is urgent need for new modern and state-of-the-art antiviral

21 strategies to be developed. 


\section{Introduction}

2 The viral family Flaviviridae comprises the genera Flavivirus, Pestivirus and

3 Hepacivirus and includes numerous important human and animal pathogens (Nulf et al.,

4 2004). The small, enveloped virions of the different members of the Flaviviridae family

5 contain a single-stranded, positive-sense RNA genome of about 9.5-12.5 kb. The

6 genome consists of a single, long open reading frame (ORF), which is flanked by 7 untranslated regions (UTRs) at the $5^{\prime}$ and $3^{\prime}$ ends. Recent studies on 8 sub-genomic Pestivirus and Flavivirus RNA replicons have revealed that the 9 non-structural (NS) proteins, which are encoded by the C-terminal part of the 10 polyprotein, play a crucial role in viral RNA replication (Nulf et al., 2004). Accordingly,

11 these proteins are assumed to form replication complexes in conjunction with genomic

12 RNA and possibly with other cellular factors.

13 Classical Swine Fever virus (CSFV) is a member of the Flaviviridae viral family affects

14 livestock and therefore represents a very important pathogen (Calisher \& Gould, 2003).

15 Even though CSFV may not be a zoonotic disease that is capable of infecting humans,

16 the consequences of its epidemics are devastating, since livestock is closely related

17 with the economical and social wellbeing of many countries in the world (Behrens et al.,

18 2004). To date no antiviral agent is available nor is expected soon. There are quite a few

19 vaccines currently available in the market for some strains of CSFV, but reportedly the

20 virus is becoming quite persistent and even under extensive vaccination there are many

21 cases of reoccurrence of the infection upon vaccination (Wu et al., 2004). Consequently,

22 there is urgent need for new antiviral strategies to be developed. In this direction, the

23 non-structural NS3 viral helicase constitutes an ideal target for structural studies 
1 towards the establishment of a comprehensive in silico platform for de novo inhibitor

2 design. The viral helicase is responsible for the unwinding of the viral genetic material

3 (Phoon et al., 2004). Thus the viral NS3 helicase is a key enzyme for the survival, 4 propagation, proliferation and finally transmission of the virus. Sequence alignments of 5 the Classical Swine Fever viral helicase identified several conserved sequence motifs 6 that are important for biological functions. So far, the crystal structures of helicases from 7 various RNA viruses have been determined, including the helicases from Hepatitis C 8 virus, Dengue virus, Yellow Fever Virus and Kunjin virus (Diana \& Bailey, 1997).

9 Herein, the 3D model of the CSFV helicase structure has been established, using 10 conventional homology modelling techniques and the X-ray determined 3D-structure of 11 the Hepatitis C virus helicase as template. In order to structurally improve the quality of 12 the homology model, it was subjected to exhaustive molecular dynamics simulations.

13 The reliability and viability of the helicase model as a plausible platform for structure

14 based drug design experiments was confirmed using a repertoire of in silico scoring 15 tools, including MOE, Procheck and Verify3D. It was determined that the established 3D 16 model of the helicase enzyme of CSFV exhibits all known structural motifs that unique 17 to the helicase enzymes of the flaviviridae viral family and therefore could provide the 18 platform for further drug design experiments. Towards this direction an in silico

19 structure-based drug design experiment was conducted, which led to the establishment

20 of a 3D pharmacophore model that is highly specific for the helicase enzyme of CSFV.

21 The pharmacophore model may be used in future experiments involving the high virtual 22 throughput screening of large molecular databases towards the identification of novel 23 anti-CSFV agents. 


\section{Methods}

2 Coordinate Preparation and Model Availability

3 3D coordinates were obtained from the X-ray solved, crystal structures of $\mathrm{HCV}$

4 helicases with RCSB codes: $1 \mathrm{~A} 1 \mathrm{~V}$ and $80 \mathrm{HM}$. The $8 \mathrm{OHM}$ entry is the full length, 5 unbound form of $\mathrm{HCV}$ helicase, whereas the $1 \mathrm{~A} 1 \mathrm{~V}$ entry contains the full length $\mathrm{HCV}$

6 helicase co-crystalized with a single strand of an oligonucleotide. All generated 3D 7 CSFV helicase models in this study are available via email request to the corresponding 8 author. All generated 3D homology models are available to download at: 9 http://www.bioacademy.gr/bioinformatics/csfv/index.html

11 Sequence alignment

12 The amino acid sequence of Classical Swine Fever viral helicase was obtained from the 13 GenBank database (accession no: NC_006551, entry name: Classical Swine Fever 14 virus, complete genome). Using the Gapped-BLAST (Altschul et al., 2004) through $15 \mathrm{NCBI}$ (Benson et al., 2007) the homologous species of the Hepatitis C virus helicase 16 was identified, which was used as template for the homology modelling of the Classical

17 Swine Fever viral helicase. The structure of the HCV helicase has been determined via 18 X-ray crystallography (Kim et al., 1998). The sequence alignment was done using the 19 online version of ClustalW (Thomson, Higgins \& Gibson, 1994). The alignment was 20 repeated using Hidden Markov Models and the result was the same as the one obtained 21 by ClustalW, due to the fact that there are several anchoring conserved motifs 22 throughout the alignment (Eddy, 1995). 


\section{Homology modelling}

2 The homology modelling of the Classical Swine Fever viral helicase was carried out

3 using the Modeller package (version 9.10) (Sali et al., 1995). The RCSB entries 1A1V

4 and $8 \mathrm{OHM}$ were both used as template structures. A different model was generated for

5 each one of the two available HCV crystal structure. The sequence alignment between

6 the raw sequence of the Classical Swine Fever and the full sequence of the $1 \mathrm{~A} 1 \mathrm{~V} \mathrm{HCV}$

7 helicase revealed almost $32 \%$ identity, whereas the identity percentage between the

8 Classical Swine Fever and the full sequence of 8OHM HCV helicase does not exceed

$935 \%$. The sequence alignment identity percentage between the two HCV template

10 structures is $93,33 \%$ due to the fact that these sequences originate from different HCV

11 strains. The abovementioned identity scores are on the lower boundary at which

12 conventional homology modelling techniques are applicable. The model that was based

13 on the 1A1V HCV structure was chosen to be used for the purposes of this study, due to

14 the fact that this structure file has been co-crystallized with a single-stranded

15 oligonucleotide. The homology model method of Modeller comprises the following

16 steps: First an initial partial geometry specification, where an initial partial geometry for

17 each target sequence is copied from regions of one or more template chains. Secondly,

18 the insertions and deletions task, where residues that still have no assigned backbone

19 coordinates are modelled. Those residues may be in loops (insertions in the model with

20 respect to the template), they may be outgaps (residues in a model sequence which are

21 aligned before the $\mathrm{C}$-terminus or after the $\mathrm{N}$-terminus of its template) or may be

22 deletions (regions where the template has an insertion with respect to the model). For

23 this study though outgaps have not been included in the homology modelling process. 
1 Third step is the loop selection and sidechain packing, where a collection of

2 independent models is created. Last step is the final model selection and refinement

3 one, where the final models are scored and ranked, after they have been

4 stereochemically checked with the "Protein Geometry" module for persisting errors.

5 Finally, necessary secondary structure predictions were performed using the NPS

6 (Network Protein Sequence Analysis) web-server and the GeneSilico MetaServer,

7 which confirmed the choice of the Hepatitis C virus helicase as template for this

8 study(http://npsa-pbil.ibcp.fr).

9

10 Molecular electrostatic potential (MEP)

11 Electrostatic potential surfaces were calculated by solving the nonlinear

12 Poisson-Boltzmann equation using finite difference method as implemented in the

13 PyMOL Software (Delano, 2002). The potential was calculated on grid points per side

$14(65,65,65)$ and the grid fill by solute parameter was set to $80 \%$. The dielectric

15 constants of the solvent and the solute were set to 80.0 and 2.0, respectively. An ionic

16 exclusion radius of $2.0 \AA$, a solvent radius of $1.4 \AA$ and a solvent ionic strength of 0.145

17 M were applied. Amber99 (Duan et al., 2003) charges and atomic radii were used for

18 this calculation.

20 Energy Minimization and Molecular Dynamics Simulations

21 Energy minimizations were used to remove any residual geometrical strain in each

22 molecular system, using the Charmm27 forcefield as it is implemented into the Gromacs

23 suite, version 4.5.5 (Hess et al., 2008). All Gromacs-related simulations were performed

24 though our previously developed graphical interface (Sellis et al., 2009). Molecular 
1 systems were then subjected to unrestrained Molecular Dynamics Simulations (MDS)

2 using the Gromacs suite, version 4.5.5 (Hess et al., 2008). MDS took place in a SPC

3 water-solvated, periodic environment. Water molecules were added using the truncated

4 octahedron box extending $7 \AA$ from each atom. Molecular systems were neutralized with

5 counter-ions as required. For the purposes of this study all MDS were performed using

6 the NVT ensemble in a canonical environment, at $300 \mathrm{~K}$ and a step size equal to 2

7 femtoseconds for a total 100 nanoseconds simulation time. An NVT ensemble requires

8 that the Number of atoms, Volume and Temperature remain constant throughout the

9 simulation.

11 Model evaluation

12 Evaluation of the model quality and reliability in terms of its 3D structural conformation is

13 very crucial for the viability of this study. Therefore, the produced models were initially

14 evaluated within the Gromacs package by a residue packing quality function, which

15 depends on the number of buried non-polar side chain groups and on hydrogen

16 bonding. Moreover, the suite PROCHECK (Laskowski et al., 1996) was employed to

17 further evaluate the quality of the produced Classical Swine Fever virus helicase model.

18 Verify3D (Eisenberg et al., 1997) was also used to evaluate whether the model of

19 Classical Swine Fever virus helicase is similar to known protein structures. Finally, the

20 Molecular Operating Environment (MOE) suite was used to evaluate the 3D geometry of

21 the models in terms of their Ramachandran plots, omega torsion profiles, phi/psi angles,

22 planarity, C-beta torsion angles and rotamer strain energy profiles. 


\section{Structure-based drug design}

3 The design of the new series of molecules via structure-based drug design was

4 conducted using Ligbuilder (version 1.2). This program will start from a "seed"

5 compound that must be manually positioned in the 3D conformational space of the

6 molecular system (Figure 6). For the purposes of this study, the mercaptoethanol

7 co-crystallized compound that was attached to the Cys431 residue of the HCV structure

8 was used as seed structure. The structures of CSFV and HCV helicase were

9 structurally superimposed and their coordinates recalculated within the same Cartesian

10 molecular system. First the S-S bond between the attached compound on the Cys431

11 and the Cys431 was broken and the hydrogens were restored from where they were

12 missing. Then the oxygen was removed, since its existence would significantly reduce

13 the number of fragments suitable for that seed-receptor arrangement. The remaining

14 compound was used as a starting point for the "growing" algorithm of LigBuilder. The

15 complex was energetically minimized using a molecular mechanics algorithm, having

16 fixed the backbone of the protein. The detached compound was entered to be the

17 starting point of the drug design algorithm and thus it was expected that this moiety

18 would be present in this position on all the new compounds, as it was already known

19 from the HCV crystal structure that it was capable of interacting with the Cys431 amino

20 acid. The algorithm used the mercaptoethanol compound as a starting point and started

21 to grow structures by combining different chemical fragments that it stores in its

22 database. The criteria are to optimally utilize the available space of the receptor and to

23 establish the maximum amount of interactions with the adjacent residues of the

24 Helicase. All the different compounds that were designed were deposited in a folder for 
1 further investigation. A similarity cut-off of $90 \%$ was used in order to make sure that

2 structurally all the different compounds in that folder would be at least $90 \%$ different.

3 The space available was filled-in with newly designed compounds, with the only 4 size-limiting parameter being the pre-defined molecular weight of the compound. After

530 generations of the Ligbuilder growing genetic algorithm, a set of 1200 compounds 6 was obtained, custom designed for the sSRNA channel of the CSFV helicase.

8 Docking

9 In order to in silico establish the complex structures of the CSVF helicase and each one 10 of the 1200 potential inhibitor compounds, the docking suite ZDOCK (version 3.0) was 11 used (Chen, Li \& Weng, 2003). Docking experiments were conducted on the models 12 that had been energetically minimized and conformationaly optimized using molecular 13 dynamics simulations. ZDOCK is a protein-protein docking suite that utilizes a 14 grid-based representation of the molecular system involved. In order to efficiently 15 explore the search space and docking positions of the molecules as rigid bodies, 16 ZDOCK takes full advantage of a three-dimensional fast Fourier transformation 17 algorithm. It uses a scoring function that returns electrostatic, hydrophobic and 18 desolvation energies as well as performing a fast pairwise shape complementarity 19 evaluation. Moreover it uses the contact propensities of transient complexes of proteins 20 to perform an evaluation of a pairwise atomic statistical potential for the docking 21 molecular system. RDOCK was utilized to refine and quickly evaluate the results 22 obtained by ZDOCK ( $\mathrm{Li}$, Chen \& Weng, 2003). RDOCK performs a fast minimization 23 step to the ZDOCK molecular complex outputs and re-ranks them according to their 24 re-calculated binding free energies. 
2 Hybrid QSAR Analysis \& Pharmacophore Elucidation

3 In this study a hybrid QSAR study was conducted using the 1200 compounds from the

4 previous drug design experiment. Since no biological activity data was available for 5 these molecules, an in silico predicted Ki score was used to rank them that was 6 primarily based on their docking scores. The aim of this analysis was initially to interpret 7 the unique characteristics of various compounds in regards to their estimated activities 8 and to provide the means required to establish a 3D-pharmacophore model that would 9 enable us to more accurately screen for anti CSFV agents.

10 We used the set of the 1200 structurally distinct inhibitor compounds, which was 11 generated by the structure based drug design algorithm. The predicted inhibitory 12 potential of those compounds, was associated with their conformations as they were 13 submitted to the Pharmacophore Elucidation Query module of MOE (Group CC, 2012).

14 The algorithm initially identifies all features common to the highest ranking compounds,

15 as they were scored by the Ligbuilder scoring algorithm, as well as features present in 16 the least ranking compounds. The first ones are retained, whereas the latter are 17 discarded. Finally, a set of regression parameters are used to estimate the activity value 18 of each compound in the training set. The relationship between the geometric fit value 19 and activity value is utilized for this computation. Pharmacophore hypotheses showing 20 best correlation in the 3D arrangement of features in a given training set compounds 21 with the corresponding pharmacological activities are formed and ranked. Several 22 structure activity relationship (SAR) pharmacophore models were derived from the 23 training set of compounds. 


\section{Results \& discussion}

2 The NS3 domain of Flaviviridae contains both the protease and the helicase coding

3 regions. For the purposes of this study, only the helicase protein of the Classical Swine

4 Fever virus was used and aligned with the Hepatitis C virus helicase structure that were 5 selected to be used as templates. Notably, all major helicase motifs, which are 6 characteristic and unique to the helicases of the flaviviridae viral family were found to be 7 completely conserved (Figure 1).

8 The 1A1V HCV helicase structure has been established by X-ray crystallography at $92.20 \AA$ resolution, while the $80 \mathrm{HM}$ at $2.30 \AA$ (Kim et al., 1998; Cho et al. 1998). The $10 \mathrm{HCV}$ helicase was selected as the most suitable template for the homology modelling of 11 the Classical Swine Fever virus. Both CSFV and HCV belong to the same viral family 12 and upon a blastp search on the Protein Databank it was found that $1 \mathrm{~A} 1 \mathrm{~V}$ and $8 \mathrm{OHM}$

13 bear the highest sequence percentage identity to CSFV (Figure 1). Furthermore, the 14 secondary structure prediction for the Classical Swine Fever virus helicase was found to 15 be very analogous to the secondary structure of the HCV helicase (data not shown).

16 More specifically, protein fold recognition techniques aim to identify and pinpoint 17 similarities among 3D protein structures that are not supplemented by significant 18 sequence similarity. The suitability of $\mathrm{HCV}$ as the best available template was also 19 confirmed by a fold recognition (FR) methodologies. The underlying principle behind FR 20 techniques is that a quick search for protein folds is made in large protein databases, 21 which is looking to identify folds that are compatible with a particular sequence. Unlike 22 simple comparisons based on sequence only, these more sophisticated methods exploit 23 all the extra 3D structural information that is readily available for many proteins. In 
1 essence these techniques turn the protein folding problem around: rather than 2 predicting how a sequence will fold, they predict how well a fold will fit a sequence

3 (Rost, Schneider \& SanderWeng, 1997). The CSVF homology modelling study 4 constitutes one of these striking examples of structurally and functionally identical 5 enzymes, which only share a low primary sequence identity. In order to confirm that $6 \mathrm{HCV}$ helicase is indeed the best choice as template we used an in-house developed 7 platform that performs protein similarity searches based on secondary structural 8 information rather than primary sequence searches.

9 The first structural superimposition between the CSFV model and its HCV template 10 exhibited an alpha-carbon RMSD that fall well within 0,64 angstroms (Figure 2). The 11 CSFV model was consequently checked with PROCHECK for its geometry 12 mathematical accuracy. In addition to that the Verify3D algorithm was employed for a 13 more in-depth evaluation for its structure. A direct compatibility comparison between the 14 3D model of Classical Swine Fever virus helicase to its own amino acid sequence was 15 performed by Verify3D. Judging strictly on location and environment, each residue is 16 assigned a structural class. In order to do this a rather large database of reference 17 structures is being used as a control. The Classical Swine Fever virus helicase model 18 scored a very reliable range between +0.33 and +0.68 . This was a further confirmation 19 in that the established CSVF 3D model is of high quality and mathematically reliable.

20 Verify3D scores that fall below the +0.1 mark are indicative of major problems in the

21 structure of the model, as it can be mathematically evaluated (Eisenberg et al., 1997).

22 The quality of the CSFV model was finally empirically confirmed in terms to its structural

23 compliance to its HCV template and all known unique characteristics of the helicases of 
1 the Flaviviridae viral family. More precisely the helicase enzymes of the flaviviridae viral

2 family belong to the helicase superfamily II, which bear seven common motifs within

3 their domains. All those motifs have been structurally conserved on the Classical Swine

4 Fever virus helicase model. They share the same 3D spatial coordinates to their

5 corresponding motifs located on the X-ray crystal helicase structures of the HCV, 6 Kunjun and Dengue viruses (Kim et al., 1998; Khromykh \& Westaway, 1997; Xu et al., 7 2005). More importantly the ssRNA interacting residues have been structurally 8 conserved on the CSFV model (Supplementary Figure 1).

10 Description of the Classical Swine Fever virus helicase model

11 As anticipated from the initial sequence alignment (Figure 1) and the consecutive 12 secondary structure prediction analysis (data not shown), the Classical Swine Fever 13 virus helicase model has retained all structural features that characterize

14 flaviviridae helicases. The GxGKT/S motif of helicase domain 1 is a Walker A motif that

15 is also conserved in kinases. Its role is to aid with the binding of the $\beta$-phosphate of ATP

16 [REF]. Evidence from mutagenesis studies confirms that any changes within this motif

17 will yield an inactive helicase. As important is the conserved DExH motif, which is

18 involved in the $\mathrm{Mg}^{2+}$ ion and ATP interaction and hydrolysis. Findings from both

19 adenylate and thymidine kinases confirm that an Aspartic acid residue is responsible for 20 the $\mathrm{Mg}^{2+}$ ion coordination that lead to a conformational layout that optimizes the ATP 21 molecule's orientation for a highly efficient nucleophilic attack (Kim et al., 1998). On the 22 other hand the QRxGRxGR motif is not associated with ATP hydrolysis rather than the 23 establishment of direct specific interactions between the helicase cleft and the nucleic 24 acid, during the unwinding process (Kim et al., 1998). Overall it was found that the 
1 CSVF helicase model has shape, size and topology identical to that of its template

2 (Figures 2 and 3). The domain layout arrangement of the model is in agreement with the

3 general flaviviridae helicase pattern. First and third domains are more closely

4 associated and are separated by a rather large channel from domain two. This channel

5 is the SSRNA cleft, where the processing of the oligonucleotide takes places and

6 unwinding occurs. During the latter process, domain two undergoes substantial

7 conformational changes. Moreover it moves away from domains one and three as the

8 strength of specific interaction in the ssRNA channel must be weakened for the

9 translocation process to occur. Figure 3 depicts the conservation of the ATP and the

10 SSRNA channels between the CSVF model and its template HCV X-ray determined

11 helicase structure (Kim et al., 1998).

SSRNA - ATP substrates and MD simulations

14 In an effort to confirm the functionality, suitability and reliability of the CSFV helicase

15 model to be used in structure-based drug design experiments, the specific interactions

16 with the ssRNA substrate were analyzed (Supplementary Figure 1). The coordinates of

17 the ssRNA molecule as well as those for the ADP molecule and the $\mathrm{Mg}^{2+}$ ions were

18 copied from the HCV template structure. Then exhaustive molecular dynamics

19 simulations were performed to the CSVF model in the presence of all substrate

20 molecules, in an explicitly solvated periodic box with SPC water molecules. Post

21 molecular dynamics analysis confirmed that the majority of the protein and the SSRNA

22 substrate interactions, are established between the backbone of the oligonucleotide and

23 a series of conserved residues on the helicase channel. Energy equilibrium is attained

24 almost simultaneously for both the model and the template helicases. The alpha carbon 
1 root mean square deviation ( ${ }^{\alpha} \mathrm{RMSd}$ ) between equivalent atoms of the HCV and the

2 post-MDs CSFV helicases does not exceed $0.37 \AA$. While large RMSd values are

3 indicative of systems of poor quality, the low RMSd values between the CSVF model

4 and the HCV template indicate that their 3D structures remain conformationally similar,

5 thus reflecting the high quality of the post molecular dynamics CSVF helicase model.

6 Moreover, the ramachandran plots of the HCV template and the CSFV model confirm

7 that there are no residues in the not allowed regions of the plot (Figure 4). The

8 Ramachandran plot is the most powerful and most established in silico tool for the

9 stereochemical evaluation of the quality of a protein's backbone, judging from its phi/psi

10 dihedral angles. The phi/psi torsion angles cluster in favorable regions of the plot for the

11 Classical Swine Fever virus helicase 3D model. None of those torsions duel in

12 disallowed regions of the plot, which correspond to residues having steric hindrance and

13 bad backbone geometries. The Classical Swine Fever virus helicase model residues

14 that are found in the allowed regions of the plot are rendered in green color, whereas

15 the generously allowed regions contain residues colored yellow. No residues are

16 located in disallowed regions of the Ramachandran plot, a fact that verifies the quality of

17 the established Classical Swine Fever virus helicase 3D model. Likewise, the dihedral

18 plots of the omega, planarity, C-beta angles and energy packing per residue are all

19 within the allowed regions, below the disallowed threshold (Supplementary Figures 2,

20 3). Taken together, all of the above confirm the viability and suitability of the homology

21 modelling of the Classical Swine Fever virus helicase model to be used in further

22 structure based in silico drug design experiments. 
1 The molecular surface of the produced Classical Swine Fever virus helicase model was

2 analyzed by calculating its electrostatic potential (Figure 5). The aim of this study was to

3 enable us to perform direct structural comparisons between the CSFV model and its

4 HCV template structures it came from. Consequently, the electrostatic potential surface

5 was calculated also for both template HCV helicase structures that were used (Figure

6 5). The CSVF model and the two helicases exhibited almost identical electrostatic

7 surfaces. More precisely, the model and its templates share all key common structural

8 features vital to viral helicases. They have a negatively charged SSRNA entrance to the

9 helicase tunnel and a clearly identical ATP hydrolysis site. Taken together these

10 similarities confirm the validity and reliability of the model, which can be used for

11 structure based drug design studies with high levels of confidence.

13 Structure based de novo drug design

14 The area surrounding the Cys431 residue was selected to be used as the target site for

15 the drug design experiment for the purposes of this study. One of the HCV crystal

16 helicase structures has been co-crystallized with a single molecule of mercaptoethanol

17 via a disuphidic bond. The Cys431 residue has established a S-S bond with a

18 mercaptoethanol $\left(\mathrm{S}-\mathrm{CH}_{4}-\mathrm{CH}_{4}-\mathrm{OH}\right)$ in the $\mathrm{HCV}$ helicase $\mathrm{x}$-ray structure. That can only

19 mean that the Cys431 is accessible to the solvent, even when ssRNA is present, and

20 could potentially be targeted for the establishment of interactions with a future inhibitor.

21 Notably, Cys431 is located in a very strategic position for the blocking of the passage of

22 the ssRNA through the helicase (Figure 6). Prior study of the HCV area surrounding the

23 Cys431 residue has indeed confirmed the ability and potential of this residue to 24 covalently bond with an HCV inhibitor and to interact with the two flanking Arginine 
1 residues (Arg393 and Arg481). The latter HCV inhibitor exerted activity in the

2 micromolar range (Kandil et al, 2009; Vlachakis et al, 2013). These residues were set to

3 define the active site of the helicase that was going to be targeted later on. The Arginine

4 residues were expected to establish $\mathrm{H}$-bonds, whereas the Cys431 residue was

5 expected to establish a S-S or an S-C bond with the inhibitor (Figure 7). Accordingly,

6 upon structural superimposition of the CSVF helicase model and the HCV crystal

7 structure it was concluded that the Arg481 position has been physicochemically

8 conserved on the CSFV helicase with a Lysine residue, while the Arg393 position has

9 been replaced with a Ser residue, which is still fully capable of establishing strong

10 hydrogen bonding interactions with potential inhibitor compounds (Figure 1 and Figure

11 11).

12 The HCV Cys431 residue constitutes an ideal target for drug design experiments not

13 just due to the fact that it had already covalently reacted with a mercaptoethanol

14 molecule in the HCV crystallization solution, but also to the fact that its position has

15 been fully conserved on the CSFV helicase too (Figure 1). More strikingly, structural

16 superposition of the Dengue helicase with the HCV and CSFV ones, revealed that the

17 Cys position is conserved on Dengue too, which belongs to the flavivirus genera.

18 Notably, HCV belongs to the hepacivirus genera and CSVF belongs to the pestivirus

19 genera. So, we have three members of the flaviviridae, all from distinct separate

20 generas that still maintain the Cys position conserved. Therefore, upon careful

21 selection of target residues on the helicases of various species within flaviviridae, there

22 is potential for the development of agents that may be active against more than one 23 helicase enzyme. 
1 Structurally, the aim was to build a bridge between the Cys431 and the Arg393 (Figure

2 7). This compound should covalently interact with the two previous residues and stand

3 in the way of the nucleic acid. In the HCV case, an ideal compound would interact with

4 the two Arg393, Arg481 and the Cys431, thus forming a bridge in the middle of the RNA

5 channel in the Helicase. Notably the physical dimensions of the selected site do not

6 change upon the molecular dynamics simulations. If the compound covalently bonds to

7 the receptor, then it is expected to be strong enough to block the passage of the ssRNA

8 thus inhibiting the Helicase (Figure 7). The lead compound designed by LigBuilder

9 expands from Arg393 through to Arg481, via Cys431 (Figure 7). It fits the available

10 accessible area of the helicase structure and interacts with even more residues than the

11 three target-residues (figure 8). Even though LigBuilder suggested it as a lead

12 compound, it is far from being considered as a lead compound, when judging from a

13 drug design point of view. This compound is only an indication of the available

14 interactions that could be potentially established between an inhibitor and the HCV

15 helicase. The process of converting the LigBuilder's compound into a viable lead from a

16 medicinal chemistry point of view is requires manual intervention of the scientist who

17 should substitute bulky groups with ones capable of establishing the same type of

18 interaction, while being simpler in structure. Figure 8, shows the lead derived from

19 LigBuilder and the interactions it establishes with the helicase. One or the most

20 important properties of an in silico designed lead compound is to be feasible to

21 synthesize. The lead must be synthesizable in order to be eventually tested for its

22 activity. This is essential in order to feedback the drug design process. Even though the

23 lead that LigBuilder generated was capable of establishing so many interactions, it had 
1 to be rejected since it would be impossible to synthesize and test such a compound in

2 the lab (Figure 6). There is a need of giving the algorithm to understand that the

3 available space is not the whole, extensive oligonucleotide channel of the helicase, but

4 is spatially defined by the three target residues.

5 That was achieved by physically incorporating a tube-like structure, which encloses all

6 key residues and defines the available space for the algorithm to work with (Figure 9).

7 The tube was originally made from carbon atoms. The PDB file of the tube was edited

8 and the atoms were converted from C (Carbon atom) to Du (Dummy atom). Du atoms

9 do not have any atomic properties and can be recognized by Ligbuilder's growing

10 algorithm as inert atoms. They are incapable of establishing interactions of any nature

11 and they have no charge. Their only function is to define the space that is available for

12 the genetic algorithm to work with. The structures that LigBuilder generated proved to

13 be a lot simpler and much more drug-like. The lead that LigBuilder proposed was

14 completely different from the one without the aid of the "tube". The custom made lead

15 was isolated and then docked again into the original receptor, where it originated from

16 (Figure 10). Only this time the "tube" was not present and the full receptor was available

17 to be explored by the docking algorithm.

18 The result of the docking confirmed that this compound, that had been specifically

19 designed for the particular area (between Arg481-Cys431-Arg393) on the helicase,

20 found its way to the suggested site and managed to establish the interactions that it was

21 expected to (Figure 10). The top ranked compound bears an extra $\mathrm{SH}_{3}$ substitution on

22 one of the phenyl rings. That $\mathrm{SH}_{3}$ moiety was able to interact with the Sulfur of the

23 Cys431 and further stabilize the docking. The stability of these compounds derives from 
1 their simplicity and rigidity. The conjugated bond and the two phenyl rings on those two

2 compounds do not allow for any flexibility. Figure 10 shows the two versions of the

3 LigBuilder lead compound with and without the $\mathrm{CH}_{4}$ substitution in one of the phenyl

4 rings. It is obvious that the presence of the extra carbon has pushed the compound a bit

5 lower, which is evidence that the extra carbon successfully established an interaction

6 with the nearby available sulphur from the Cys431 residue.

7 This way the complexity of the structure was forced to be low, by choosing small

8 molecular weights and by limiting the number of interaction between the future

9 compound and the protein to those found by the multi-fragment search. The number of

10 Hydrogen bond donors and acceptors was estimated by the Lipinski's rule of five initially

11 and later on by limiting the number of $\mathrm{H}$ bonds to the ones already acquired by the

12 multi-fragment search. This made the drug design approach using the linking algorithm

13 very fast and ensured that all new suggested compounds will be simple in structure and

14 thus synthesizable.

3D Pharmacophore Elucidation and the DNP-poly(A) substrate.

17 3D Pharmacophore designing methods take into account both the three-dimensional

18 structures and binding modes of receptors and inhibitors, in order to identity regions that

19 are favorable or not for a specific receptor-inhibitor interaction. The description of the

20 receptor-inhibitor interaction pattern is determined by a correlation between the

21 characteristic properties of the inhibitors and their biochemically determined enzymatic

22 activity.

23 Based on the findings of the structure based, de novo drug design experiment, a 3D 24 pharmacophore was generated for the active site of CSFV, as a molecular model that 
1 ensembles all steric and electronic features that are necessary to ensure optimal

2 covalent and non-covalent interactions with CSFV (Figure 11). The pharmacophoric

3 features investigated, included positively or negatively ionized regions, hydrogen bond

4 donors and acceptors, aromatic regions and hydrophobic areas. For the

5 pharmacophore elucidation process for CSFV's SSRNA channel all 1200 de novo

6 generated compounds, from the drug design experiment were used in their in silico

7 docked conformations. Moreover, a structure based 3D pharmacophore was created

8 using the SSRNA interacting residues on the CSFV helicase channel. The final

9 pharmacophore model elucidated for CSFV helicase was the result of the overlaying of

10 two different pharmacophores that were then reduced to their shared features. This way

11 only their common set of interactions was retained. Our complex-based pharmacophore

12 used a query set that represented a set of receptor-inhibitor interaction fingerprints,

13 which were in the form of docked in silico virtual inhibitor complexes.

14 It was determined that a set of criteria had to be satisfied, in order for a candidate

15 inhibitor compound to be active for the CSFV and HCV helicase. Firstly, using the HCV

16 helicase's 3D structure, there should be one electron donating group (Figure 11,

17 magenta color) in the proximity of the Arg393 residue (or the corresponding Lysine on

18 the CSFV helicase model). Moreover three electron donating groups should be present

19 in the proximity of both Arg393 and Arg481 amino acids (or the corresponding Lysing

20 and Aspartic acid on the CSFV helicase model). Those interaction sites may not strictly

21 represent hydrogen bonds but water or ion mediated bridges too. Finally the space

22 in-between Arg393 and Arg481 in the HCV or the corresponding Lys-Asp residues on

23 the CSFV helicase should be occupied by a large conjugated set of either hydrophobic 
1 or aromatic rings. Notably the physical dimensions of this site do not change on either

2 the HCV or CSFV model upon molecular dynamics simulations. Collectively, according

3 to our in silico prediction model, a potent candidate inhibitor of the CSFV helicase

4 should satisfy all of the previously described pharmacophoric features. 


\section{Conclusions}

2 The 3D model of the Classical Swine Fever virus helicase was designed using the

3 homologous X-ray crystal structure of the Hepatitis C viral helicase as template. The model

4 was successfully in silico evaluated both in terms of its geometry, fold recognition and

5 compliance to the criteria required as a member to the Flaviviridae viral family. Furthermore,

6 a comprehensive 3D pharmacophore model was constructed alongside the proposition of a

7 lead compound as an in silico predicted potential inhibitor for this enzyme. It is therefore

8 proposed that the Classical Swine Fever virus helicase model will be suitable for further in

9 silico structure-based de novo drug design experiments. Future experiments may lead to

10 the screening of large chemical compound libraries in search of a potent inhibitor. These

11 computer-based methodologies are now becoming integral part of the drug discovery

12 process that may eventually lead to the development of potential inhibitor structures against

13 the Classical Swine Fever viral helicase in the future. 


\section{References}

2

Altschul, S. F., Madden, T. L., Schäffer, A. A., Zhang, J., Zhang, Z. and Miller, W. (1997). Gapped BLAST and PSI-BLAST: a new generation of protein database search programs. Nucleic Acids Res. 25, 3389-3402.

Behrens, S.-E. , Grassmann, C. W. , Thiel, H.-J. , Meyers, G. \& Tautz, N. (1998). Characterization of an autonomous subgenomic pestivirus RNA replicon. Journal of Virology 72, 2364-2372.

Benson, D. A., Karsch-Mizrachi, I., Lipman, D. J., Ostell, J. and Wheeler, D. L. (2007). GenBank. Nucleic Acids Res. 35, 21-25.

Calisher, C. H. and Gould, E. A. (2003). Taxonomy of the virus family Flaviviridae. Adv. Virus Res. 59, 1-19.

R. Chen, L. Li, and Z. Weng, ZDOCK: an initial-stage protein-docking algorithm. Proteins 52 (2003) 80-7.

Cho, H.S., Ha, N.C., Kang, L.W., Chung, K.M., Back, S.H., Jang, S.K., Oh, B.H. (1998). Crystal structure of RNA helicase from genotype $1 \mathrm{~b}$ hepatitis $\mathrm{C}$ virus. A feasible mechanism of unwinding duplex RNA. J.Biol.Chem. 273: 15045-15052

DeLano WL (2002) The PyMOL User's Manual San Carlos, CA, USA.: DeLano Scientific.

Diana, G. Bailey, T. 1997. Compounds, compositions and methods for treatment ofhepatitis C. United States Patent No. 5,633,388

Y. Duan, C. Wu, S. Chowdhury, M.C. Lee, G. Xiong, W. Zhang, R. Yang, P. Cieplak, R. Luo, T. Lee, J. Caldwell, J. Wang, and P. Kollman, A point-charge force field for molecular mechanics simulations of proteins based on condensed-phase quantum mechanical calculations. J Comput Chem 24 (2003) 1999-2012.

Eddy SR. Multiple alignment using hidden Markov models. Proc Int Conf Intell Syst Mol Biol. 1995;3:114-20

D. Eisenberg, R. Luthy, and J.U. Bowie, VERIFY3D: assessment of protein models with three-dimensional profiles. Methods Enzymol 277 (1997) 396-404

Group CC (2012) Molecular Operating Environment (MOE). 1010 Sherbrooke St. West, Suite 910, Montreal, Canada, H3A 2R.

B. Hess, C. Kutzner, D. van der Spoel and E. Lindahl, GROMACS 4: algorithms for highly efficient, load-balanced, and scalable molecular simulation. J. Chem. Theory Comput. 4 (2008) 435-447.

Kandil S, Biondaro S, Vlachakis D, Cummins AC, Coluccia A, Berry C, Leyssen P, Neyts J and Brancale A. Discovery of a novel HCV helicase inhibitor by a de novo drug design approach. Bioorg Med Chem Lett. 2009 Jun 1;19(11):2935-7

Khromykh, A. A. \& Westaway, E. G. (1997). Subgenomic replicons of the flavivirus Kunjin: construction and applications. Journal of Virology 71, 1497-1505. 
1 Kim, J. L., Morgenstern, K. A., Griffith, J. P., Dwyer, M. D., Thomson, J. A., Murcko, M. A., Lin, C. and

2 Caron, P. R. (1998). Hepatitis C virus NS3 RNA helicase domain with a bound oligonucleotide: the crystal

3 structure provides insights into the mode of unwinding. Structure 6, 89-100.

4 R.A. Laskowski, J.A. Rullmannn, M.W. MacArthur, R. Kaptein, and J.M. Thornton, AQUA and 5 PROCHECK-NMR: programs for checking the quality of protein structures solved by NMR. J Biomol NMR $68(1996)$ 477-86.

7 L. Li, R. Chen, and Z. Weng, RDOCK: refinement of rigid-body protein docking predictions. Proteins 53 8 (2003) 693-707.

9 Nulf, C. J. and Corey, D. 2004. Intracellular inhibition of hepatitis C virus (HCV)internal ribosomal entry 10 site (IRES)-dependent translation by peptide nucleic acids (PNAs) and locked nucleic acids (LNAs). 11 Nucleic Acids Research 32(13), pp. 3792-3798.

12 Phoon, C. Ng, P. Ting, A. Yeo, S. Sim, M. 2001. Biological Evaluation of Hepatitis C Virus Helicase 13 Inhibitors. Bioorganic \& Medicinal Chemistry Letters (11) pp 1647-1650

14 Rost, B., Schneider, R., Sander, C. Protein Fold Recognition by Prediction-based Threading. J. Mol. Biol. 15 (1997) 270, 471-480

16 Sali A, Potterton L, Yuan F, van Vlijmen H, Karplus M (1995) Evaluation of comparative protein modeling 17 by MODELLER. Proteins 23: 318-326.

18 D. Sellis, D. Vlachakis, and M. Vlassi, Gromita: a fully integrated graphical user interface to gromacs 4. 19 Bioinform Biol Insights 3 (2009) 99-102.

20 The NPS (Network Protein Sequence Analysis) web-server: http://npsa-pbil.ibcp.fr

21 Thompson, J. D., Higgins, D. G. and Gibson, T. J. (1994). CLUSTAL W: improving the sensitivity of 22 progressive multiple sequence alignment through sequence weighting, position specific gap penalties and 23 weight matrix choice. Nucleic Acids Res. 22, 4673-4680.

24 Vlachakis D, Koumandou VL, Kossida S. A holistic evolutionary and structural study of flaviviridae 25 provides insights into the function and inhibition of HCV Helicase. PeerJ. 2013; 1 :e74

26 Xu, T., Sampath, A., Chao, A., Wen, D., Nanao, M., Chene, P., Vasudevan, S.G., Lescar, J. (2005).

27 Structure of the Dengue virus helicase/nucleoside triphosphatase catalytic domain at a resolution of 2.4

28 A. J.Virol. 79: 10278

29 Wu, J., Bera, A. K., Kuhn, R. J. and Smith, J. L. (2005). Structure of the flavivirus helicase: implications for 30 catalytic activity, protein interactions, and proteolytic processing. J. Virol. 79, 10268-10277. 


\section{Figure Legends}

2

Figure 1. Primary sequence alignment of CSVF against the HCV template (RCSB entry: $1 \mathrm{~A} 1 \mathrm{~V}$ ). All seven major conserved motifs of Flaviviridae helicases have been highlighted in red color. The key conserved amino acids that will be used as targets for the drug design steps have been highlighted in bold and magenta color.

Figure 2. The CSFV helicase homology model. A: Ribbon representation of the produced Classical Swine Fever virus helicase model. B: the Classical Swine Fever virus helicase model superimposed with its Hepatitis $C$ virus helicase template (RCSB entry: $1 \mathrm{~A} 1 \mathrm{~V})$. The Classical Swine Fever virus helicase model is in red, whereas the Hepatitis $C$ virus helicase template is in green and the ssRNA substrate is in blue.

Figure 3. Structural location of the conserved motifs. The Hepatitis $C$ virus helicase (in green color) is structurally superposed over the Classical Swine Fever virus helicase model (colored in Red). The major motifs have been color-coded according to the conventions of Fig. 1, and are showing in space filling representation. The ssRNA oligonucleotide is showing in blue ribbon representation. The white arrow point to the ATP site of the helicase. Notably, the conserved motifs sit around the ATP site, where they coordinate the ATP hydrolysis by the helicase enzyme.

Figure 4. The Ramachandran plots for the HCV template structure (PDB entry: 1A1V) on the top and the CSFV model after the molecular dynamics simulations below.

Figure 5. Molecular electrostatic potential surfaces. Electrostatic surfaces potential for the Classical Swine Fever virus helicase model and the Hepatitis $C$ virus helicase template (X-ray structure: $1 \mathrm{~A} 1 \mathrm{~V})$.

Figure 6. The seed structure for the drug design experiment. This is a mercaptoethanol molecule that has been co-crystalized on the HCV helicase crystal structure. Choosing the right starting point for the growing algorithm is very influential to the algorithm. Here the seed was included in the PDB file (1A1V).

Figure 7. The dimensions of the ssRNA channel of HCV. Distances and the available space in the Helicase' $s$ active site. All newly designed compounds should have dimensions that could be accommodated in the above showing area and interact with Arg393 and one or both of the Cys431 and Arg481 residues.

Figure 8. Lead compound from LigBuilder docked into the helicase. The resulting molecule from the drug design experiment contains 14 chiral centers and is therefore impossible to synthesize. It had to be reduced to a manageable synthetically molecule that still maintains most of the original interactions with the helicase enzyme. 
1 Figure 9. Drug design using the tube spatial restrain. The Application of the "tube"

2 structure that simplified the task of LigBuilder, by limiting the available 3D

3 conformational space than new compounds can occupy.

4

5

6

Figure 10. The in silico designed lead compounds. A-B: The two lead compounds positioned in the SSRNA channel of the HCV helicase. These compounds were designed by using LigBuilder and the "tube". C: The mode of interaction between the best, most promising LigBuilder compound and the three target residues of the HCV ssRNA channel.

Figure 11. The Pharmacophore model for the HCV template and the CSFV model. A: the HCV template with the bound oligonucleotide (white ribbon). B: The 3D pharmacophore in the presence of the key $\mathrm{HCV}$ residues (in ball and stick representation) and the corresponding CSFV amino acids (in wire representation). The pharmacophore color coding is blue for hydrogen acceptor, green for hydrophobic or aromatic rings and magenta for hydrogen donors. C: The final pharmacophore model for the CSFV helicase.

Suppl. Figure 1. All residues surrounding the ssRNA fragment, color-coded as Figure 2. The sSRNA interacting regions are almost identical between the Classical Swine Fever virus helicase model and the Hepatitis $C$ helicase template.

Suppl. Figure 2. The dihedral (omega, planarity and C-beta) and energy plots for the $\mathrm{HCV}$ template structure (PDB entry: 1A1V). A: The plot is showing the omega torsion profile. B: The profiles of phi/psi angles. C: The planarity the third the C-beta torsion angle profile. D: the rotamer strain energy profile in $\mathrm{kcal} / \mathrm{mol}$. The $\mathrm{HCV}$ helicase sequence is on the $\mathrm{X}$-axis.

Suppl. Figure 3. The dihedral (omega, planarity and C-beta) and energy plots for the CSFV homology model after the molecular dynamics simulations. A: The plot is showing the omega torsion profile. B: The profiles of phi/psi angles. C: The planarity the third the C-beta torsion angle profile. D: the rotamer strain energy profile in $\mathrm{kcal} / \mathrm{mol}$. The CSVF helicase sequence is on the $X$-axis. 


\section{Figure 1}

Primary sequence alignment of CSVF against the HCV template (RCSB entry: 1A1V)

All seven major conserved motifs of Flaviviridae helicases have been highlighted in red color. The key conserved amino acids that will be used as targets for the drug design steps have been highlighted in bold and magenta color.

$\begin{array}{ll}\text { CSF } & \text { KITTMNRGEFRITLAT GAGKTTELPRSVIEEIGRHKRVLVIPLRAAAESVYQYMRQKH } \\ \text { HCV } & \text { PPAVPQSFQVAHLHAPTGSGKSTKVPAAYAAQ-G--YKVLVLNPSVAATLGFGAMSKAH } \\ \text { CSF } & \text { PSIAFNLRIGEMKEGDMATGITYASYGYFCQMPQPKLRAAMVEYSFIFLDEYHCATPEQL } \\ \text { HCV } & \text {-GVDPNIRTG-VRTITTGSPITYSTYGKF--LADGGXSGG--AYDIIICDECHSTDATSI } \\ \text { CSF } & \text { AIMGKIHRFSE--NLRVVAM-TATPAGTVTTTGQKHPIEEFIAPEVMKGEDLGSEYLDIA } \\ \text { HCV } & \text { LGIGTVLDQAETAGARLVVLATATPPGSVTV---PHPNIEEVALST-TGE------IPFY } \\ \text { CSF } & \text { GLKIPVEEMKSNM-LVFVPTRNMAVETAKKLKAKGYNSGYYYSGEDPSNLRVVTSQSPYV } \\ \text { HCV } & \text { GKAIPLEVIKGGRHLIFCHSKKKCDELAAKLVALGINAVAYYRGLDVS---VIPTSGDVV } \\ \text { CSF } & \text { VVATNAIESGVTLPDLDVVVDTGLKCEKRI--RLSPKMPFIVTGLKRMAVTIGEQAQRRG } \\ \text { HCV } & \text { VVATDALFTG-----DFDSVIDCNTCVTQTVDFSLDPTFTIETTTLPQDAVS--RTQRRG } \\ & \\ \text { CSF } & \text { RVGRVKPGRYYRSQETPVGSKDYHYDLLQAQRYGIEDGINITKSFREMNYDWSLYEED-- } \\ \text { HCV } & \text { RTGRGKPG-IYRFVAP--GERPSGMFDSSV-LCECYDAGXA-WYELTPAETTVRLR-AYM } \\ \text { CSF } & \text {---SLMITQ--LEILNNLLISEELPMAVKNIMARTDHPEPIQLAYNSYETQVPVLFPKIK } \\ \text { HCV } & \text { NTPGLPVCQDHLEFWEGVFT--LTHIDAHFLSQTKQSGENFPYLVAYQATVCA-----R } \\ & \\ \text { CSF } & \text { NGEVTDSYDNY--TFLNARKLGDDVPPYVYATEDEDLAVELLGLDWPDPGNQGTVEA } \\ \text { HCV } & \text { AQAPPPSWDQMWKCLIRLKPTLHGPTPLLYRLGAVQNEVT---LTHPITKYIMTCMS }\end{array}$




\section{Figure 2}

The CSFV helicase homology model.

A: Ribbon representation of the produced Classical Swine Fever virus helicase model. B: the Classical Swine Fever virus helicase model superimposed with its Hepatitis C virus helicase template ( RCSB entry: $1 \mathrm{~A} 1 \mathrm{~V}$ ). The Classical Swine Fever virus helicase model is in red, whereas the Hepatitis $\mathrm{C}$ virus helicase template is in green and the ssRNA substrate is in blue .

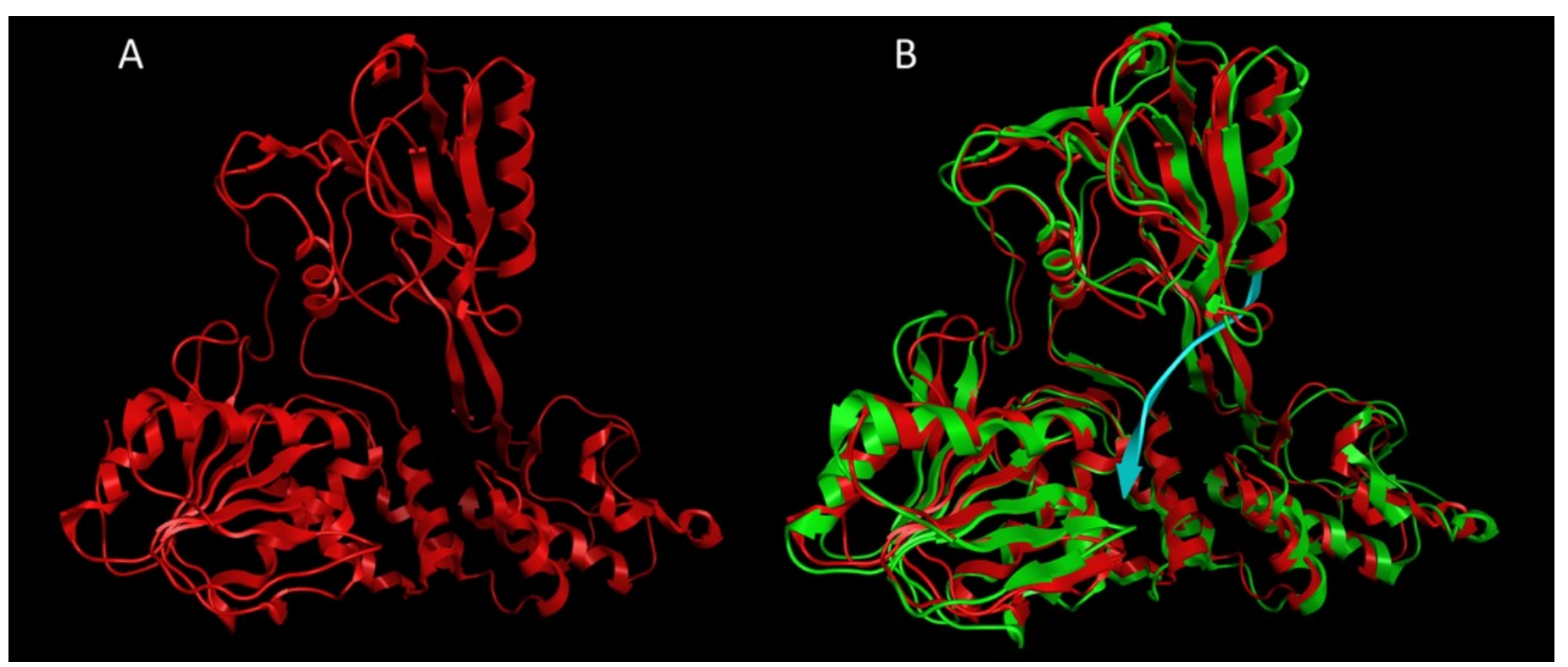




\section{Figure 3}

Structural location of the conserved motifs.

The Hepatitis C virus helicase (in green color) is structurally superposed over the Classical Swine Fever virus helicase model (colored in Red). The major motifs have been color-coded according to the conventions of Fig. 1, and are showing in space filling representation. The ssRNA oligonucleotide is showing in blue ribbon representation. The white arrow point to the ATP site of the helicase. Notably, the conserved motifs sit around the ATP site, where they coordinate the ATP hydrolysis by the helicase enzyme.

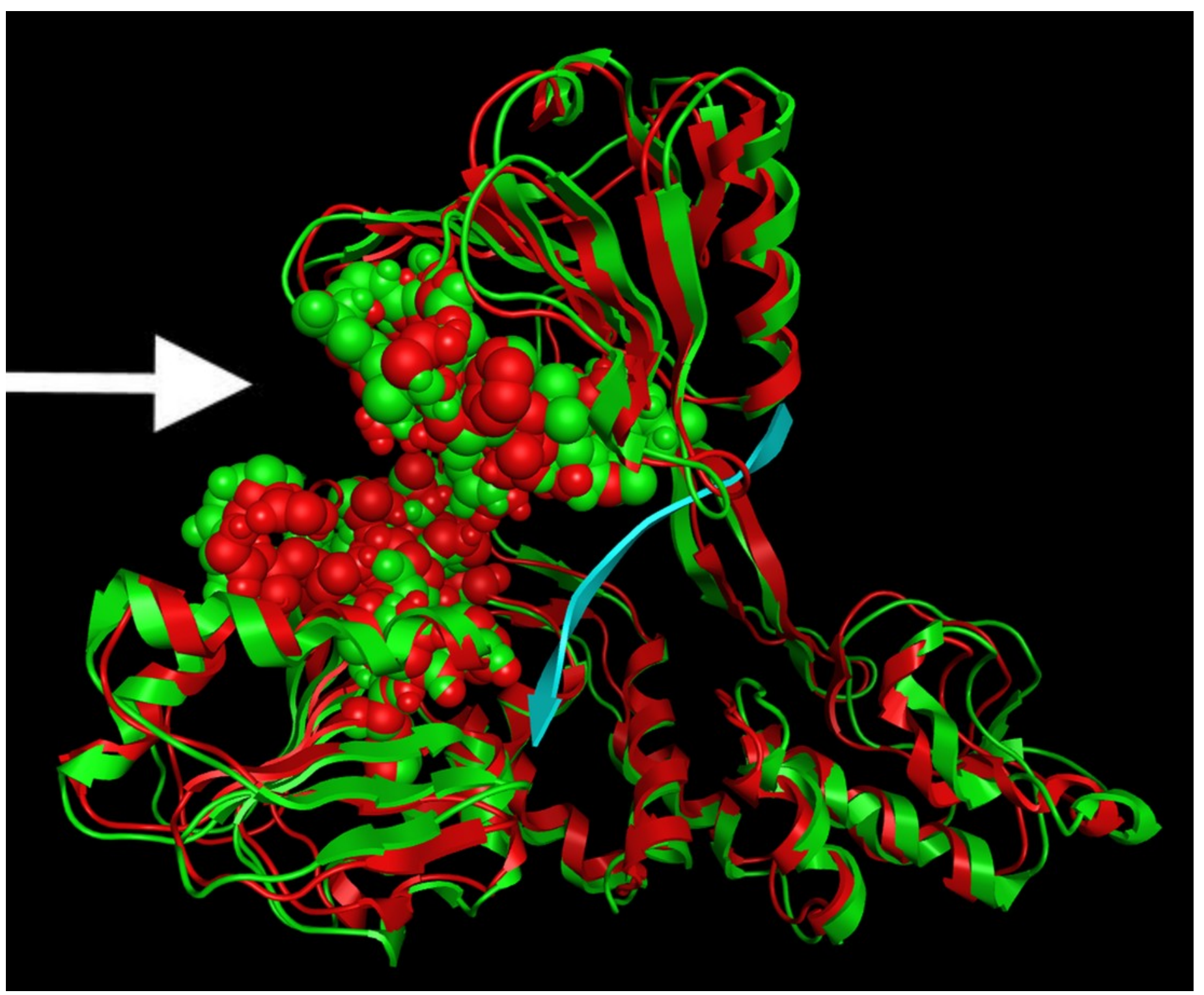




\section{Figure 4}

The Ramachandran plots for the HCV template structure (PDB entry: 1A1V) on the top and the CSFV model after the molecular dynamics simulations below 


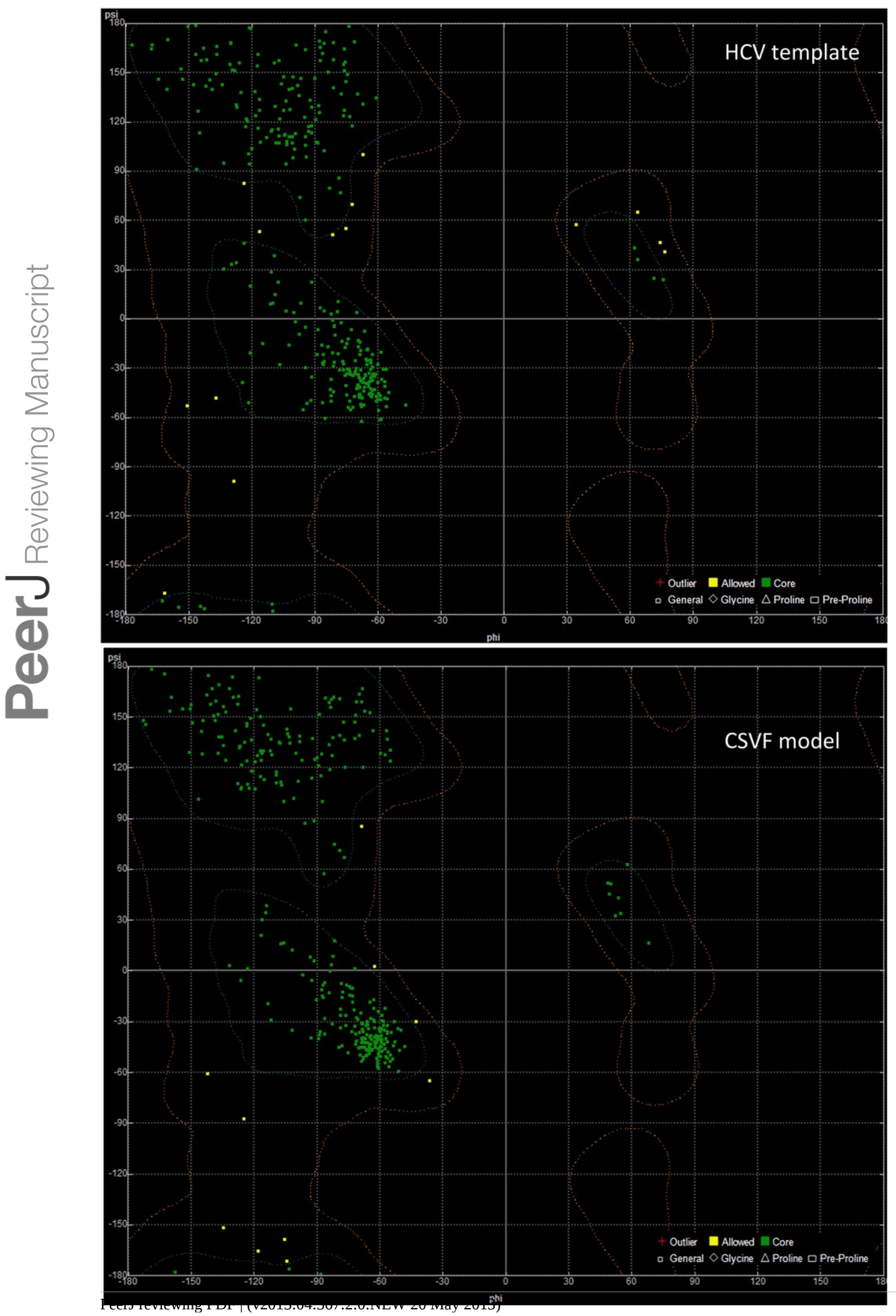




\section{Figure 5}

Molecular electrostatic potential surfaces.

Electrostatic surfaces potential for the Classical Swine Fever virus helicase model and the Hepatitis C virus helicase template (X-ray structure: $1 \mathrm{~A} 1 \mathrm{~V})$.

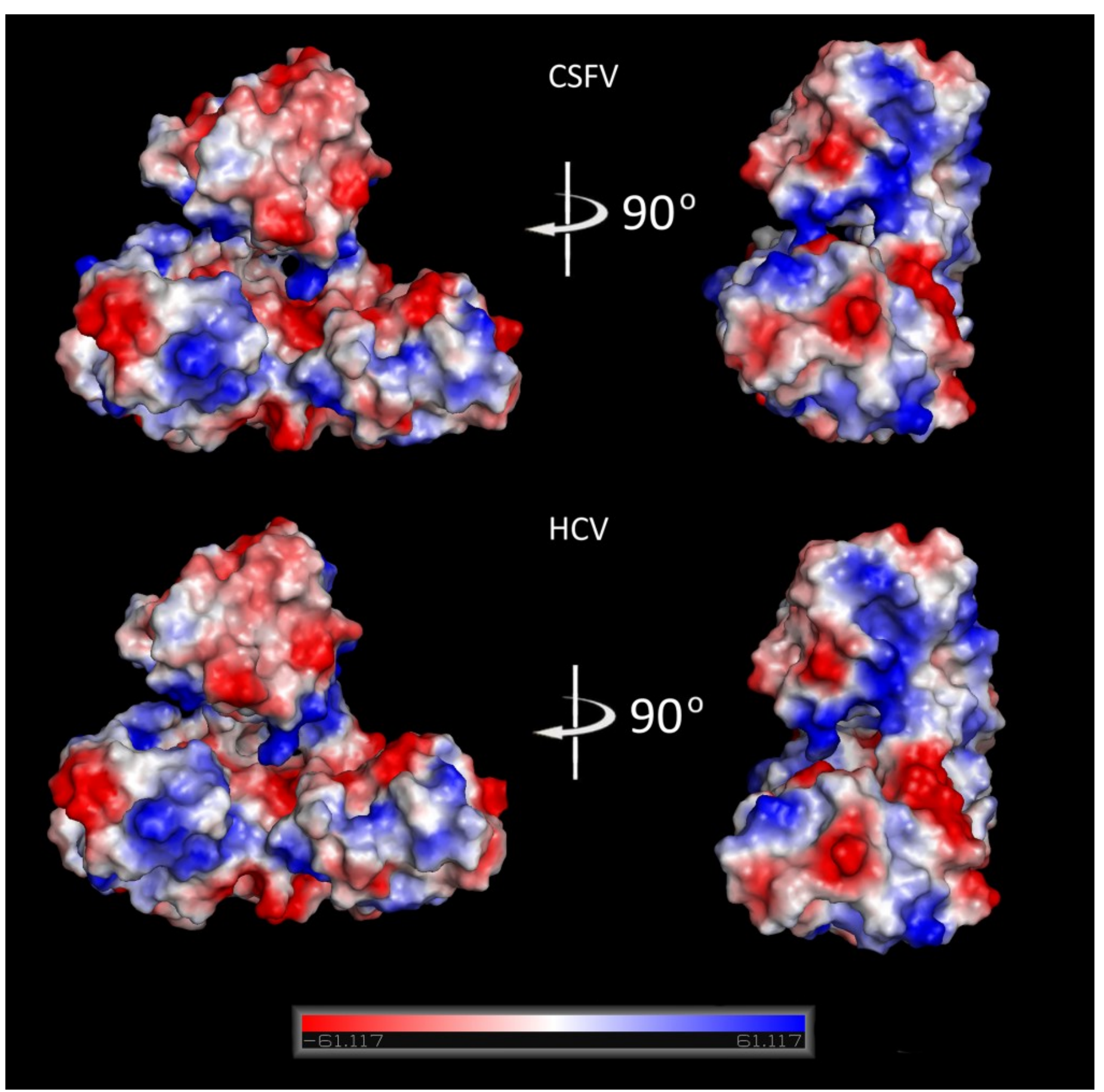




\section{Figure 6}

The seed structure for the drug design experiment.

This is a mercaptoethanol molecule that has been co-crystalized on the HCV helicase crystal structure. Choosing the right starting point for the growing algorithm is very influential to the algorithm. Here the seed was included in the PDB file (1A1V).
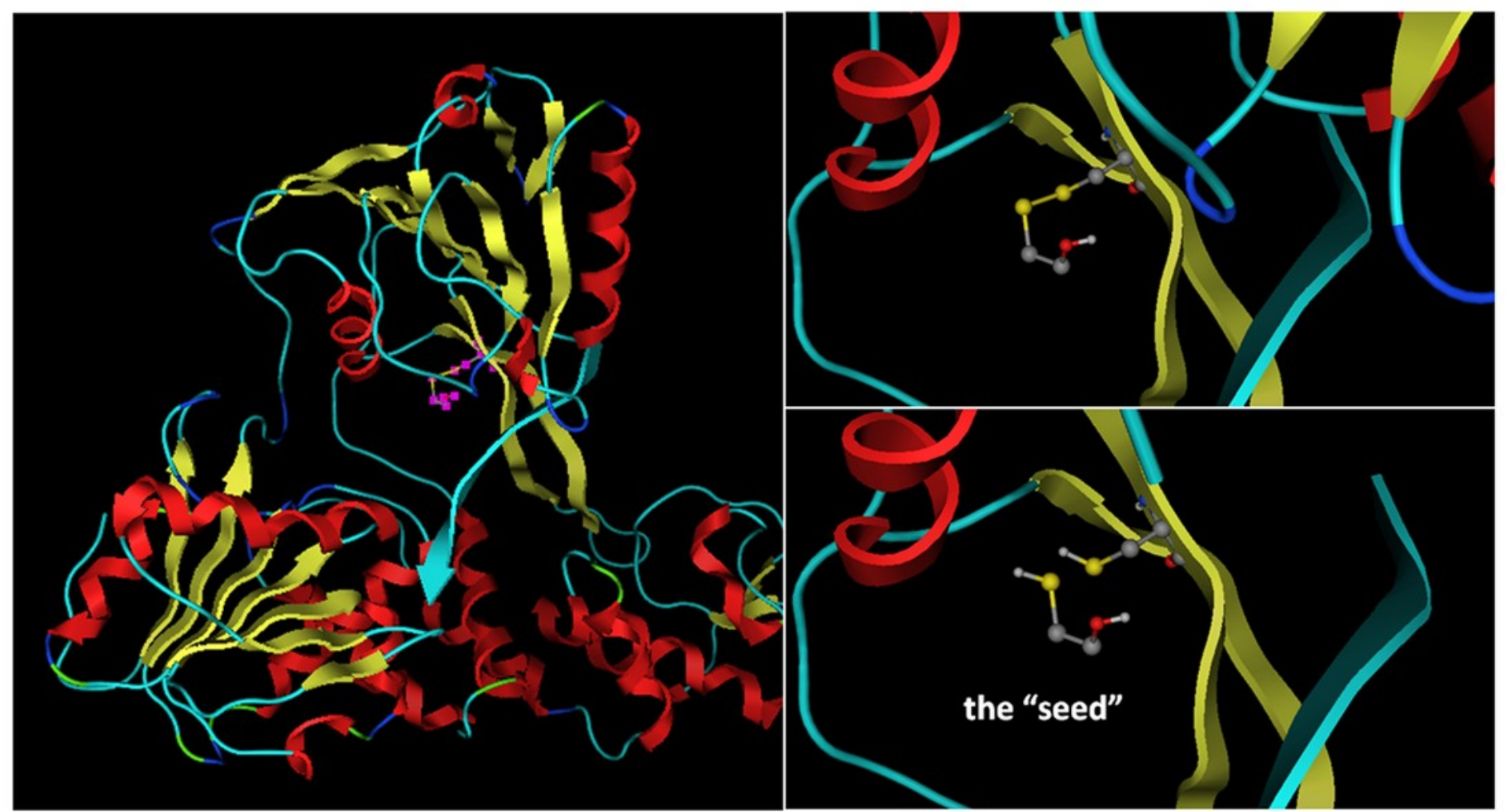


\section{Figure 7}

The dimensions of the ssRNA channel of HCV. Distances and the available space in the Helicase' s active site.

All newly designed compounds should have dimensions that could be accommodated in the above showing area and interact with Arg393 and one or both of the Cys431 and Arg481 residues.

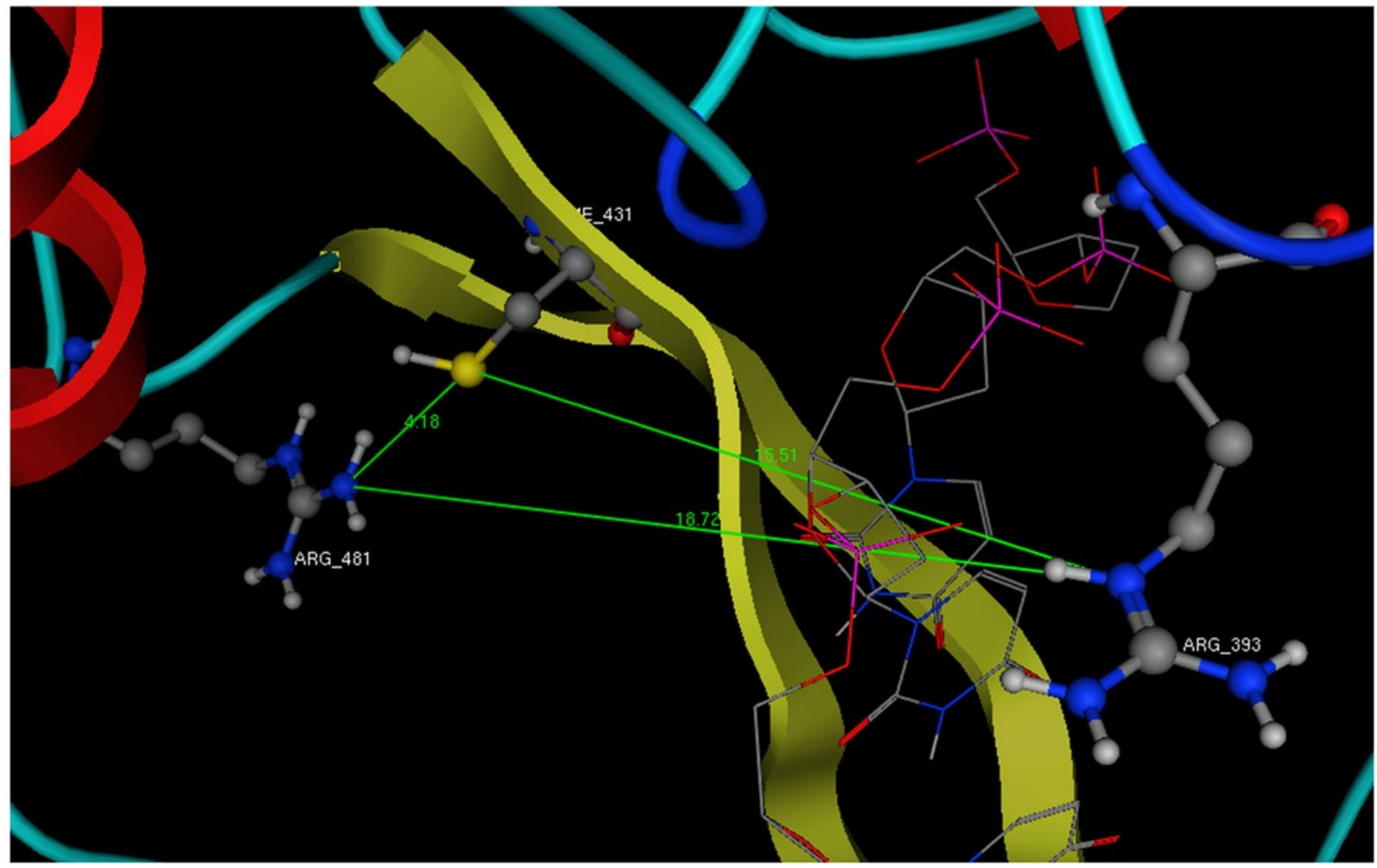




\section{Figure 8}

Lead compound from LigBuilder docked into the helicase.

The resulting molecule from the drug design experiment contains 14 chiral centers and is therefore impossible to synthesize. It had to be reduced to a manageable synthetically molecule that still maintains most of the original interactions with the helicase enzyme.

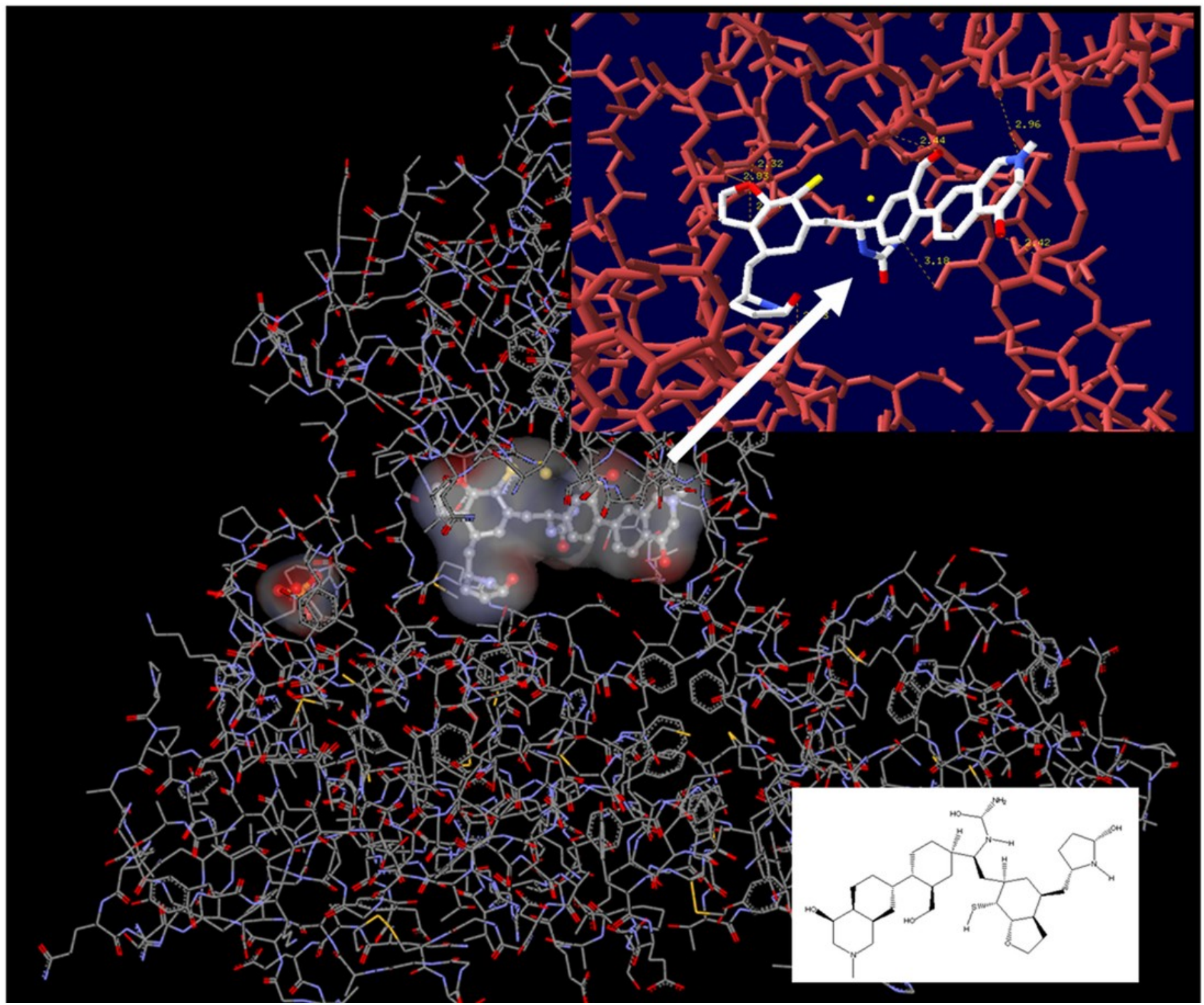




\section{Figure 9}

Drug design using the tube spatial restrain.

The Application of the "tube" structure that simplified the task of LigBuilder, by limiting the available

3D conformational space than new compounds can occupy.

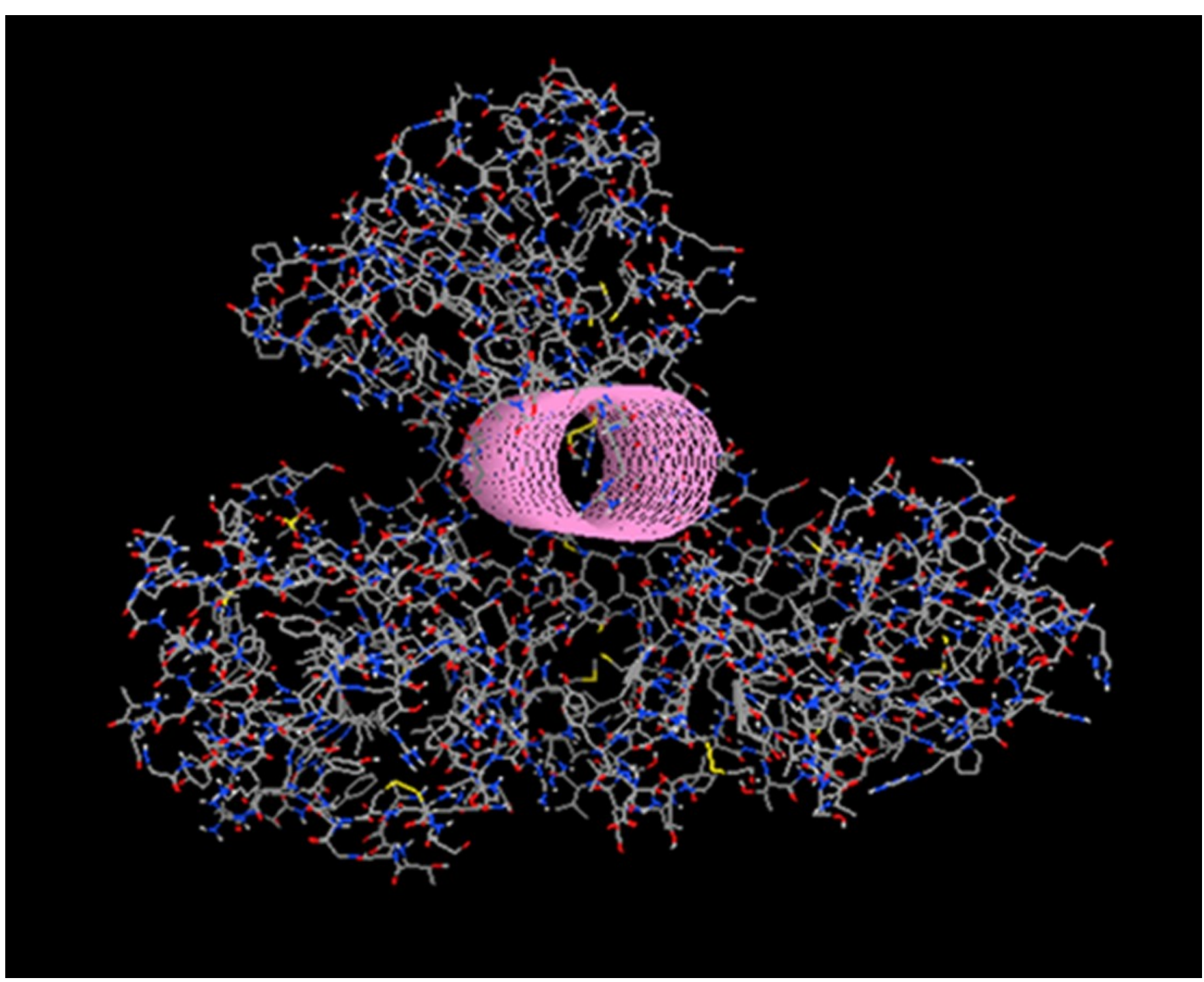




\section{Figure 10}

The in silico designed lead compounds.

A-B: The two lead compounds positioned in the ssRNA channel of the HCV helicase. These compounds were designed by using LigBuilder and the "tube". C: The mode of interaction between the best, most promising LigBuilder compound and the three target residues of the HCV ssRNA channel.
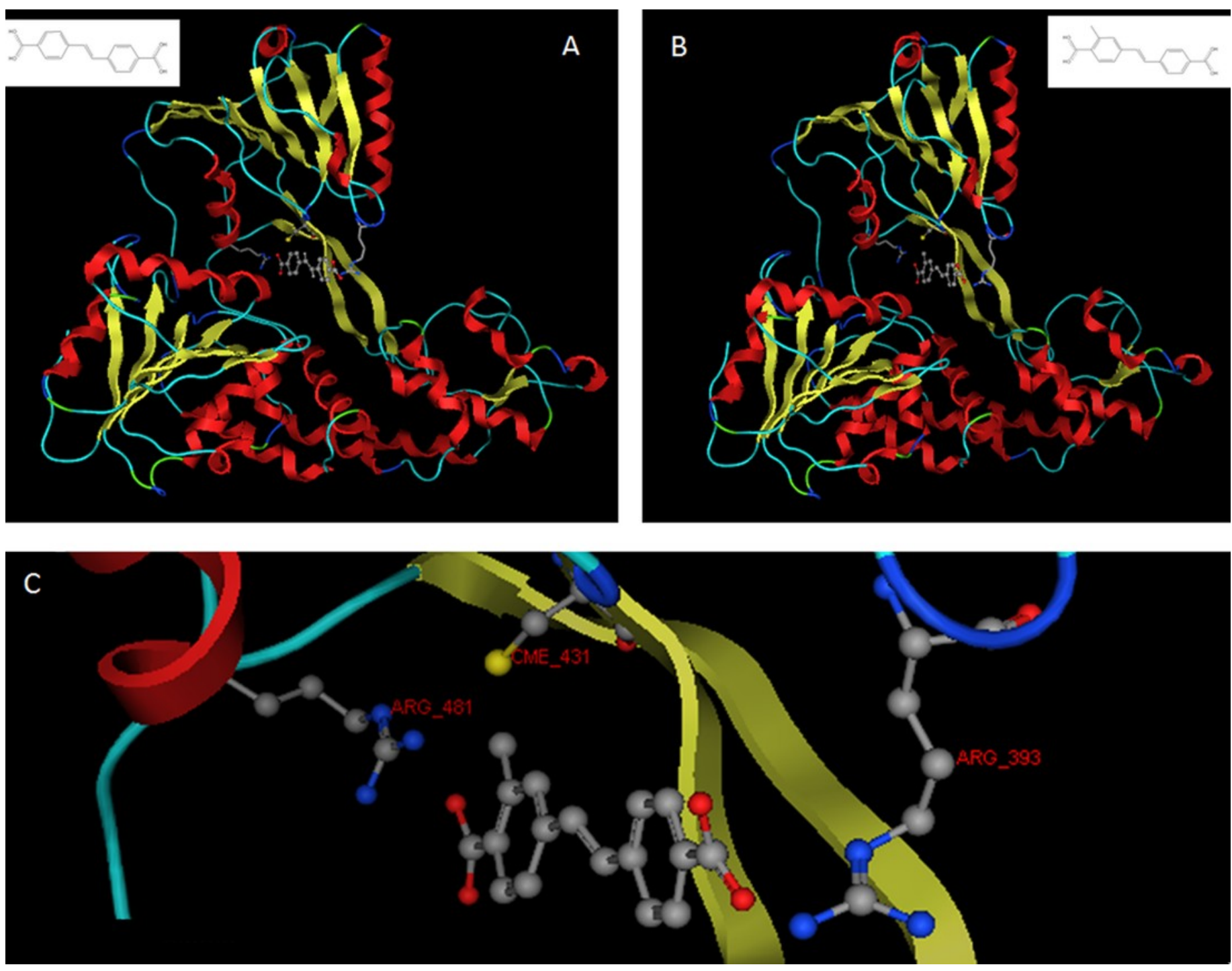


\section{Figure 11}

The Pharmacophore model for the HCV template and the CSFV model.

A: the HCV template with the bound oligonucleotide (white ribbon). B: The 3D pharmacophore in the presence of the key HCV residues (in ball and stick representation) and the corresponding CSFV amino acids (in wire representation). The pharmacophore color coding is blue for hydrogen acceptor, green for hydrophobic or aromatic rings and magenta for hydrogen donors. C: The final pharmacophore model for the CSFV helicase
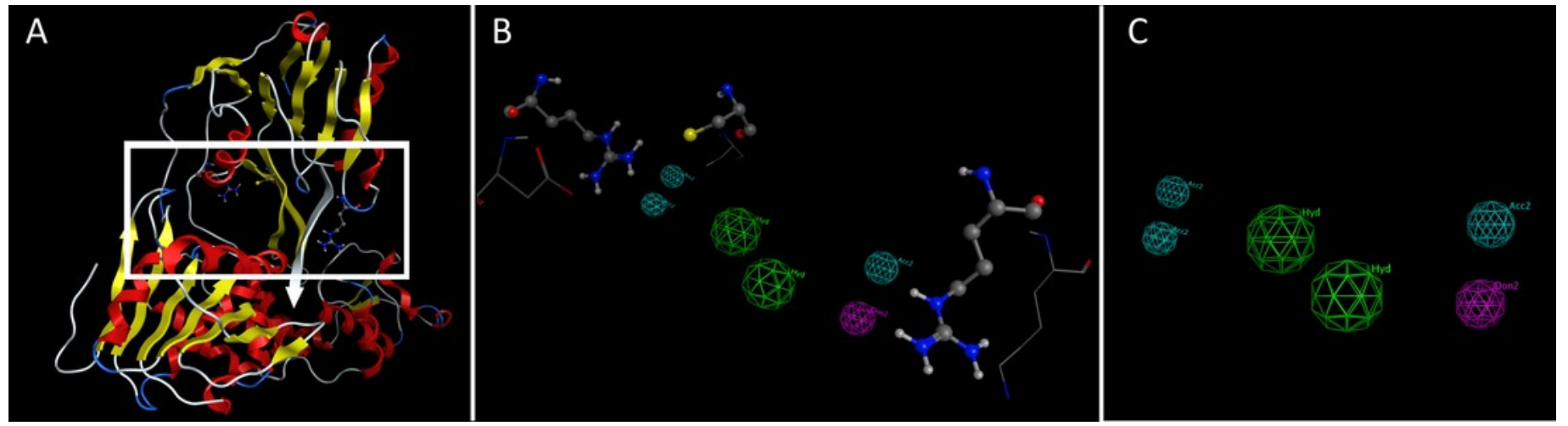